\title{
artigo
}

Dias, A.P.O.; Campagnoli, M.; Meneguetti, C.; Ramos, M.J.; Maria Silva, E.;

Práticas de enfermagem durante a pandemia de COVID-19: relato de experiências

\section{Práticas de enfermagem durante a pandemia de COVID-19: relato de experiências}

\author{
Nursing practices during the COVID-19 pandemic: experiences report \\ Prácticas de enfermería durante la pandemia COVID-19: informe de experiencias
}

\begin{abstract}
RESUMO
Objetivo: Analisar e refletir sobre as práticas de enfermagem durante a pandemia da COVID-19 em serviços de saúde públicos e privados. Método: Estudo descritivo, qualitativo de relato de experiências baseado nas práticas assistenciais e gerenciais de enfermeiras que trabaIham em cidades dos Estados de São Paulo e Minas Gerais-Brasil. Realizaram-se encontros semanais nos meses de outubro e novembro de 2020 e foram discutidas as vivências relacionadas ao enfrentamento à pandemia, buscando-se elucidar os aspectos centrais das práticas da enfermagem. Resultados: Os temas foram organizados em três categorias: Gestão de cuidados, envolvendo o gerenciamento ea assistência prestada; Educação em saúde, permeando o dia a dia com novos conhecimentos; e Sofrimento mental, os impactos na saúde desses profissionais. Conclusões: Considera-se que a pandemia interferiu na prática de enfermagem, na atuação dos profissionais e no modo de produzir saúde. Salientamos a necessidade da educação permanente em saúde ser implementada nos serviços.
\end{abstract}

DESCRITORES: Prática Profissional; Cuidados de Enfermagem; Infecções por Coronavirus; Educação Continuada.

\section{ABSTRACT}

Objective: To analyze and reflect on nursing practices during the COVID-19 pandemic in public and private services. Method: A descriptive, qualitative report of experiences developed based on assistential and management practices from nurses who worked in public or private health services in the State of São Paulo and Minas Gerais/Brazil. Weekly meetings were conducted in October and November, 2020, when we discussed experiences related to confronting the pandemic seeking to list the common of its nursing practices. Results: The reports were organized into three categories: Care management, involving management and assistance provided; Health education, permeating daily life with new knowledge; and Mental suffering health, the consequences on these professionals' health who are working in these health services. Conclusions: The pandemic is considered to have interfered with nursing practice, the performance of professionals and the way of producing health. We emphasize the need for continuing education to be implemented in health services.

DESCRIPTORS: Professional Practice; Nursing Care; Coronavirus Infections; Continuing Education.

\section{RESUMEN}

Objetivo: Analizar y reflexionar sobre las prácticas de enfermería durante la pandemia COVID-19 en los servicios públicos y privados. Método: Reporte descriptivo, cualitativo de experiencias basadas en las prácticas de atención y gestión de enfermeras que laboran en ciudades de los Estados de de São Paulo y Minas Gerais-Brasil. Se realizaron reuniones semanales en los meses de octubre y noviembre de 2020 y se discutieron las experiencias relacionadas con el afrontamiento de la pandemia, buscando elegir los puntos de convergencia del desempeño de las enfermeras. Resultados: Los informes fueran organizados en tres categorías: Gestión de la atención, que involucra la gestión y la assistência brindada; Educación sanitária, impregnando la vida diaria de descubrimientos y nuevos conocimientos; y Sufrimiento mental, los reflejos sobre la salud de los profesionales que trabajan en estos servicios de salud. Conclusiones: Se considera que la pandemia ha interferido con la práctica de enfermería, el desempeño de los profesionales y la forma de producir salud. Destacamos la necesidad de que la educación permanente se implemente en los servicios de salud. DESCRIPTORES: Práctica Profesional; Atención de Enfermería; Infecciones por Coronavirus; Educación Continua.

RECEBIDO EM: 30/01/2021 APROVADO EM: 17/02/2021

\section{Ana Paula de Oliveira Dias}

Enfermeira, pós-graduada (lato sensu) em urgência e trauma, mestranda do Programa de Pós-Graduação em Ciências da Saúde da Universidade Estadual de Campinas (Unicamp).

ORCID: 0000-0002-2577-8113 


\section{Michele Campagnoli}

Enfermeira, gestora da atenção primária em saúde, doutoranda do Programa de Pós-Graduação em Ciências da Saúde da Unicamp. ORCID: 0000-0002-7874-2296

\section{Carolina Meneguetti}

Enfermeira, pós graduada (lato sensu) em saúde do adulto e idoso, aluna especial da disciplina EG 138 do Programa de Pós-Graduação em Ciências da Saúde da Enfermagem da Unicamp.

ORCID: 0000-0002-7209-7652

\section{Maria José Ramos}

Enfermeira, pós graduada (lato sensu) em Estratégia Saúde a Família e terapia intensiva com ênfase em cardiologia, aluna especial da disciplina EG 138 do Programa de Pós-Graduação em Ciências da Saúde da Enfermagem da Unicamp.

ORCID: 0000-0001-7862-1884

\section{Eliete Maria Silva}

Enfermeira, livre docente, professora associada na Faculdade de Enfermagem da Unicamp.

ORCID: 0000-0001-7549-2677

\section{INTRODUÇÃO}

A $s$ práticas de enfermagem são desenvolvidas como cuidado e são executadas e adaptadas de acordo com a situação e as necessidades presentes. De forma que o conhecimento se transforma na aplicação, não perdendo a essência, e ganhando em diversificação na execução da prática ${ }^{1}$.

No contexto das práticas de enfermagem, compreender sua forma de organização e sua razão significa compreender muito mais do que as práticas de enfermagem em si, mas assume a dimensão de dar significado a diferentes sujeitos - individuais e coletivos - que se interconectam nos diversos cenários nos quais atuam os profissionais de saúde ${ }^{2}$.

A análise das práticas requer que se compreenda a organização do poder e do conhecimento em determinado contexto e que se estabeleça sua relação com os diversos dispositivos sociais, políticos e econômicos. Com isso, analisar as práticas auxilia a compreender, tanto como os arranjos são estabelecidos, quanto como são admitidos e aceitos em um determinado momento ${ }^{2}$.

$\mathrm{O}$ ano de 2020 foi marcado pela pandemia de COVID-19, causado por um novo coronavírus (2019-nCoV) que foi detectado pela primeira vez em Wuhan, na China, no final do ano de $2019^{3}$.

Denominada de COVID-19 a doença causada pelo SARS-Cov-2, já contaminou mais de 9 milhões de brasileiros, tendo uma incidência de $4.647,0$ por 100 mil habitantes, contabilizando 237.489 óbitos, possuindo uma taxa de letalidade de $2,4 \%$ e uma taxa de mortalidade de 113,0 por 100 mil habitantes ${ }^{4}$.

Em relação aos serviços estudados, eles se localizam em São Paulo com 44,04 milhões de habitantes, apresenta 1.901.74 casos confirmados de COVID-19, 55.971 óbitos, taxa de incidência $4.141,1$ por 100 mil habitantes e taxa de mortalidade 121,9 por 100 mil habitantes ${ }^{4}$. E no estado de Minas Gerais com aproximadamente 20,87 milhões de habitantes, possui 798.711 casos confirmados de COVID-19, 16.595 óbitos, taxa de incidência $3.773,1$ por 100 mil habitantes e taxa de mortalidade 78,4 por 100 mil habitantes ${ }^{4}$.

Com o espectro clínico não completamente definido o tratamento tem sido de suporte e inespecífico ${ }^{5}$. O manejo clínico da síndrome respiratória na COVID-19 consiste inicialmente na oxigenoterapia, antibioticoterapia empírica, monitorização dos sinais vitais e sinais de complicações clínicas como insuficiência respiratória, sepse e choque séptico. Assim como a prevenção de complicações e agravos por meio da anticoagulação profilática ${ }^{6}$.

Para o enfrentamento desta pandemia, os profissionais de enfermagem atuam no tratamento, prevenção, detecção e recuperação dos doentes, atuando na porta de entrada e em todo o conjunto dos serviços de saúde, configurando-se juntamente com os demais profissionais da saúde na linha de frente, no combate à COVID-19 ${ }^{7}$.

Dada a magnitude e o impacto dessa pandemia no sistema de saúde brasileiro se faz necessário o compartilhamento de experiências assistenciais e gerenciais da prática da enfermagem diante desse desafio mundial, que gera e tem gerado aprendizados, perdas, incertezas, medos e mudanças. Muitos são os questionamentos, mas optamos por aprofundar em apenas um: "Qual o impacto da pandemia de COVID-19 nas práticas cotidianas de enfermagem?"

Sendo assim, este estudo teve o objetivo de analisar e refletir sobre as práticas de enfermagem durante a pandemia de COVID-19 em serviços públicos e privados de municípios do interior de Minas Gerais e São Paulo.

\section{METódo}

Trata-se de um estudo descritivo, qualitativo, do tipo de relatos de experiências no qual descreveu aspectos vivenciados pelas autoras, em suas práticas de enfermagem nos seguintes serviços: centro de saúde, unidade de pronto atendimento, unidade de atendimento ambulatorial (atendimento domiciliar e telemonitoramento em saúde) e unidade de terapia intensiva, pertencentes às cidade de Campinas - SP, Limeira - SP e Poços de Caldas - MG. 
As vivências baseadas nas práticas de enfermagem foram explanadas durante encontros virtuais semanais, por meio da plataforma Zoom e Google Classroom, que ocorreram em outubro e novembro de 2020, e estas vivências estavam relacionadas ao início da pandemia em março de 2020 até o período dos encontros.

$\mathrm{O}$ critério de inclusão foi associar as mudanças cotidianas que permeavam as práticas de enfermagem de todas as profissionais envolvidas em seus diversos serviços e excluiu se a especificidade de cada serviço.

O estudo teve como pergunta norteadora: Qual o impacto da pandemia de COVID-19 nas práticas cotidianas de enfermagem? As experiências foram expostas à reflexão coletiva de treze enfermeiros (especialistas, mestrandas, mestres, doutorandos e doutora), apoiada na autoanálise das práticas de enfermagem e na problematização do cotidiano vivenciado durante a pandemia.

Para a construção do relato, seguiu-se o método da narrativa, baseado na escrita de cada autora sobre sua vivência, construindo uma única narrativa, destacando-se as convergências marcantes na sua prática cotidiana ${ }^{8}$.

Por meio destas reflexões das descrições das práticas de cada uma, houve a geração de novas ações, novas reflexões e nova sistematização contribuindo na atuação de cada serviço e na disposição para a sistematização deste relato.

\section{RESULTADOS}

As práticas de enfermagem permeiam o dia a dia dos profissionais de saúde conforme o desenvolvimento do trabalho e se diversificam de acordo com o serviço de saúde, existindo similaridades e peculiaridades entre eles.

Organizamos os relatos de experiências em três categorias: Gestão do cuidado, no qual descrevemos a organização dos serviços e transformação do dia a dia do trabalho dos profissionais; Educação em saúde no qual foi descrito o percurso dos trabalhadores para obtenção das informações e conhecimentos, assim como os treinamen- tos em serviço; e o Sofrimento mental, demonstrando os diversos sentimentos e anseios da equipe de saúde.

\section{Gestão do cuidado}

Devido a pandemia foi necessária a organização do fluxo de atendimento e assistência prestada aos pacientes em cada instituição de acordo com as demandas e características dos serviços. Foram estabelecidos diversos protocolos e várias modificações conforme a descoberta e divulgação de informações sobre a COVID-19. A diminuição dos atendimentos programados e o início do teleatendimento em saúde, foi novidade para a prática profissional e trouxe consigo diversos desafios.

Os serviços modificaram também o perfil da comunidade atendida, como por exemplo a unidade de pronto atendimento que passou a ser hospital de referência para os casos de COVID-19, atendendo pacientes por meio da regulação regional e não mais pacientes por demanda espontânea, o que pode ter gerado aumento da demanda nos centros de saúde e nas outras unidades da rede de urgência e emergência do município.

Além disso, a gestão dos recursos humanos foi impactada pelos afastamentos dos profissionais que eram do grupo de risco e por aqueles que adoeciam, intensificando a crise do dimensionamento de profissionais aptos a trabalhar na linha de frente somado aos atendimentos clínicos e de urgência e emergência, uma vez que não houve interrupção das atividades rotineiras dos serviços.

Paralelamente haviam os apelos pelas melhores condições de trabalho da categoria profissional que mesmo com o reconhecimento e homenagens presentes na mídia não eram suficientes para garantir sua autonomia, valorização, carga horária de trabalho adequada, proteção e salários dignos.

A gestão de materiais inicialmente se relacionava a oferta dos Equipamentos de Proteção Individual (EPI) sendo necessário realizar controle da oferta destes, assim como treinamentos para a utilização adequada. Posteriormente, foi necessário o gerenciamento de equipamentos como monitores, ventiladores, máscaras não reinalantes, assim como algumas medicações que começaram a escassear.

A comunicação entre os membros da equipe, da gestão com a equipe e da equipe com a população, facilitou no planejamento e organização das atividades e aumentou a segurança no desenvolvimento das práticas profissionais. Por outro lado, em alguns serviços essa comunicação não foi tão eficaz, os profissionais eram surpreendidos pelas normas impostas pela gestão sem adequada comunicação, participação e envolvimento da equipe.

Percebe-se que a gestão do cuidado foi construída dia a dia para atender as demandas existentes em cada serviço e precisou ser revista diariamente.

\section{Educação em saúde}

$\mathrm{Na}$ prática do enfermeiro assistencial e gestor, é possível identificar algumas potencialidades, como educação em saúde, liderança, autonomia e comunicação dentro da equipe de saúde, ressalta-se a importância desse profissional na articulação do cuidado e como mediador da equipe de saúde.

As capacitações foram desenvolvidas de diversas maneiras para aplicabilidade dos protocolos e proteção individual dos profissionais. Percebeu-se que os profissionais buscaram conhecimentos fora de seu local de trabalho, por meio de cursos e leituras de artigos científicos. Os profissionais tiveram espaços coletivos para discussão e troca de experiência ou compartilhamento de novos saberes, facilitando sua prática no dia a dia.

Em um dos serviços foi realizada uma ação no território para conscientização da população das prevenções e precauções sobre a COVID-19, mas esta ação foi pontual, devido à escassez de recursos humanos. Ações como esta devem ser contínuas para impactar efetivamente no processo saúde e doença. Houve comunicação com o Conselho Local de Saúde para compartilhar informações sobre o território e sanar dúvidas trazidas pela população, eles são o elo entre a unidade de saúde e a população.

Existe uma necessidade de atualização 
contínua do conhecimento, o qual transforma a prática profissional por meio da reflexão de cada profissional e coletivamente. Sendo as práticas transformadas na reflexão da própria prática. Além disso, a pandemia colocou a necessidade de aprender a aprender como algo vital.

\section{Sofrimento mental}

A pandemia influenciou na prática da enfermagem no que diz respeito tanto à sua organização quanto à sua execução. Notou-se que foram vários os desafios percorridos, como baixos salários, carga horária de trabalho excessiva, dimensionamento inadequado, falta de valorização profissional, meios de comunicação limitados e a questão do sofrimento mental dos profissionais.

Muitas informações e transformações, assim como descobertas, entendimentos, medos, angústias, dúvidas, preocupações, insegurança, tristeza, solidão, incerteza, tensão e estresse. Tudo isso circulou o trabalho da equipe e de cada profissional em seu atendimento. Gerou sofrimento e refletiu nas práticas do profissionais.

Além disso, houve o afastamento de alguns profissionais, seja por "burnout" ou devido a contaminação pelo SAR-Cov-2, configurando-se em uma doença laboral que ultrapassa os limites de doença individual ressoando em toda a família do profissional, associado ao medo, angústia e preocupações.

A forma que os profissionais viram para reduzir tal ansiedade e medos foi o diálogo entre eles, mas não foi o suficiente para prevenir o desenvolvimento da fadiga, estresse e demais sintomas que abalam psicologicamente os trabalhadores.

\section{DISCUSSÃO}

Durante o enfrentamento de uma pandemia, além da infraestrutura e da reconfiguração do papel dos hospitais e unidades de saúde na perspectiva de atender as demandas, emerge o eixo do trabalho cotidiano dos profissionais de saúde, sobretudo enfermeiros, técnicos e auxiliares de enfermagem, que representam expressiva parcela da força de trabalho em saúde no país. Destaca-se nesse trabalho o papel do enfermeiro que desenvolve de forma articulada atividades assistenciais, gerenciais e de educação em saúde ${ }^{9}$.

Dentre os inúmeros desafios pontuados no contexto da pandemia, nos deparamos com a gestão de pessoas, do serviço e do cuidado, como importante aspecto de nossa prática profissional.

Observamos que os profissionais da enfermagem demonstraram angústia, ansiedade, depressão e estresse, pela desinformação e por não conseguir controlar ou manejar adequadamente a situação vivenciada ${ }^{10}$.

A supervisão de enfermagem deve subsidiar as práticas da equipe, detectar fragilidades, compartilhar conhecimentos, manter firmeza nas ações, com empatia, para mobilizar recursos para enfrentamento de um momento atípico, como este da pandemia. Podendo contribuir para o manejo do estresse, medo e insegurança da equipe 9 .

Outro ponto abordado com frequência pelos profissionais de enfermagem é a questão da desvalorização e sobrecarga de trabalho que vai ao encontro dos apelos da categoria por condições e jornadas de trabalho, piso salarial digno e plano de aposentadoria especial e à valorização do trabalho ${ }^{7}$.

Em um estudo realizado em 2018, que teve como objetivo compreender as potencialidades e desafios no desenvolvimento de melhores práticas de enfermagem diferentes contextos assistenciais, podemos observar que alguns pontos identificados como desafios foram: equipe reduzida, remuneração baixa, falta de diálogo entre enfermagem e equipe multiprofissional, ausência de Educação Permanente em Saúde (EPS), pouco apoio (da gestão), interferência política na assistência, sobrecarga de trabalho e falta de valorização e cuidado com cuidador, o que demonstra algumas similaridades com nosso relato de experiência ${ }^{11}$.

Em relação a potencialidades para melhores práticas de enfermagem apontadas pelos enfermeiros que participaram do mesmo estudo, foram identificados: trabalho em equipe, liderança, vínculo com o paciente, espaços de aprendizagem coletiva e empoderamento do enfermeiro ${ }^{11}$.

Foi possível observar o relevante papel da educação permanente na prática da enfermagem. Pelos relatos, verificou-se a necessidade de capacitações dos protocolos instituídos e também a busca de novos saberes e conhecimentos através de leituras e discussão de casos entre as equipes, exemplos esses que convergem com o exposto em estudo sobre os principais desafios da enfermagem no enfrentamento da COVID-19 sob a perspectiva de enfermeiros gestores na macrorregião oeste de Santa Catarina ${ }^{12}$.

A contribuição da educação permanente, aliada ao uso de protocolos e da Prática Baseada em Evidências (PBE), para desenvolver a resiliência da força de trabalho de enfermagem e para a autonomia da profissão, alicerçada na utilização e incorporação do processo de enfermagem e em consonância com suas raízes teóricas ${ }^{12}$.

A educação dos profissionais de saúde sobre a COVID-19 não contribui apenas para orientar os pacientes/usuários, mas como parte de seu processo formativo de implementação da PBE em seu cotidiano acompanhando a contínua atualização técnico científica ${ }^{13}$ e atuando ativamente no controle das informações e notícias falsas disseminadas sobretudo nas mídias sociais ${ }^{14,15}$.

Outro assunto colocado em pauta, foi a questão da saúde mental dos profissionais de enfermagem. É um profissional que sente medo, vibra com a melhora do paciente, sonha com melhores condições de saúde, mas que, em tempos de pandemia, frequentemente se angustia e teme as incertezas do que está por vir ${ }^{16}$.

Nas análises dos depoimentos de profissionais de enfermagem evidenciou-se que, se por um lado, carregam um sentimento de impotência e frustração, expressando a vulnerabilidade do profissional de enfermagem diante das adversidades para as quais não há respostas, por outro lado evidenciam a visão tradicional e determinista do cuidar/cuidado enquanto 


\section{artigo}

Dias, A.P.O.; Campagnoli, M.; Meneguetti, C.; Ramos, M.J.; Maria Silva, E.

Práticas de enfermagem durante a pandemia de COVID-19: relato de experiências

reflexo de um saber cartesiano que precisa encontrar respostas favoráveis para todas as situações e promover a cura a qualquer preço, considerando que a sociedade não está preparada para "perder" ${ }^{16}$.

A pandemia trouxe significativo impacto para as práticas de enfermagem, trazendo diversas mudanças e reorganização na forma de prestar o cuidado, que pode ser considerado o principal objeto de trabalho da enfermagem. Essas mudanças não devem ficar estagnadas, precisam ser reformuladas diariamente, uma vez que o processo saúde doença e cuidado é dinâmico, temporal, cultural e possui relação direta e estreita com as desigualdades sociais, culturais e econômicas do país.

\section{CONCLUSÃO}

Ao discutir características que definem as práticas de enfermagem no cenário da atual pandemia, notamos a diversidade existente nas mesmas e suas potências, reconhecidas por meio das estratégias adotadas, como o planejamento, educação permanente, no qual a protagonista é a equipe de saúde em parceria com a gestão e a sociedade.

Também observamos as fragilidades, como a sobrecarga do trabalho, o esgotamento físico e mental. Ressaltamos a necessidade de cuidarmos dos profissionais da linha de frente, e contribuir para sua segurança e capacidade de atuação com a implementação da supervisão e da educação permanente nas instituições de modo a lidar e aprender sempre com os aspectos que são afetados no dia a dia do trabalho.

Considera-se portanto que a pandemia interferiu potencialmente na prática de enfermagem, na atuação dos profissionais e no modo de produzir saúde, evidenciando os desafios e potencialidades dos profissionais de enfermagem, que assumem o protagonismo, a mediação e a realização do cuidado, em um contexto de crise. Cabe ressaltar a capacidade de resiliência e reinvenção dos profissionais e a importância dessa força de trabalho para a sociedade.

\section{REFERÊNCIAS}

1. Rocha JSY, Almeida MCP. O saber de enfermagem e sua dimensão prática. $2^{a}$ ed. São Paulo. Ed. Cortez, 1989.

2. Velloso ISC, Ceci C, Alves M. Reflexões sobre relações de poder na prática de enfermagem. Rev. Gaúcha Enferm., Porto Alegre (RS). 2010, jun; 31 (2): 388-91.

3. Organização Pan-Americana da Saúde / Organização Mundial da Saúde. Alerta Epidemiológico Complicações e sequelas da COVID-19. 12 de agosto de 2020, Washington, D.C.: PAHO/ WHO; 2020

4. Brasil. Ministério da Saúde. Painel de casos de doença pelo coronavírus 2019 (COVID-19) no Brasil pelo Ministério da Saúde. Última atualização 01/01/2021, às 19h. Disponivel em: https:// covid.saude.gov.br/. Acesso em 13 de fevereiro de 2021.

5. Brasil. Ministério da Saúde. Secretaria de Atenção Especializada à Saúde Departamento de Atenção Hospitalar, Domiciliar e de Urgência. Protocolo de Tratamento do Novo Coronavírus (2019-nCoV). $1^{\text {a }}$ edição. Brasília - DF. 2020. Disponivel em: https://portaldeboaspraticas.iff.fiocruz.br/wp-content/ uploads/2020/03/Protocolo-de-manejo-clinico-para-o-novo-coronavirus-2019-ncov.pdf. Acesso em 17 de novembro de 2020.

6 Organização Mundial da Saúde. Manejo Clínico da COVID-19 - Orientação Provisória - 27 de maio de 2020. Disponivel em: https://www.who.int/publications/i/item/ clinical-management-of- covid-19.

7. Fortes ECN, Pires DEP. Os apelos da enfermagem nos meios em tempos de coronavírus. Rev Bras Enferm. 2020; 73 (Suppl2): e20200225.

8. Sá MC, Miranda L, Diniz DS, Savi ESA, Teixeira ES, Fonseca MLG. Oficinas clínicas do cuidado: efeitos da narratividade sobre o trabalho em saúde. Ed Fiocruz, RJ, 2019, pág. 51 à 60.
9. Chaves LDP, Fabro GCR, Galiano C, Trovó MC, Tomaz WB, Gleriano JS. Reflexões acerca do exercício da supervisão de enfermagem no enfrentamento da covid-19. CuidArte, Enferm ; 14(1): 10-17, 2020.

10. Silva, CG et al. Enfermagem e "estar com" em um mundo com Covid-19: um olhar existencialista. Rev Gaúcha Enfermagem. 2021.

11. Weber ML, Vendruscolo C, Adamy EK, et al Melhores práticas em enfermagem na rede de atenção à saúde: potencialidades e desafios. 2019;9:e3504. DOI: http://dx.doi.org/10.19175/recom. v9i0.3504).

12. Geremias DS, Vendruscolo C, Celuppi IC, Adamy EK, Toso BRGO, Souza JB. 200 Years of Florence and the challenges of nursing practices management in the COVID-19 pandemic. Rev. Latino-Am. Enfermagem. 2020; 28: e3358.

13. Rodrigues NH, Silva LGA. Gestão da pandemia Coronavírus em um hospital: relato de experiência profissional. J. nurs. health. 2020;10(n.esp.):e20104004.

14. Posseti J, Boncheva K. Desinfodemia: decifrar a desinformação sobre a COVID-19. Módulo 1. Organização das Nações Unidas para a Educação, a Ciência e a Cultura. 2020. Disponível em:https://pt.unesco.org/covid19/disinfodemic. Acesso em 13 Fev. 2021

15. Motta LD, Freitas AA, Janovik Júnior RX, Blatt CR, Caregnato RCA. COVID-19 evidências para todos: desenvolvimento de um objeto de aprendizagem no ensino em saúde [preprint] Rev Gaúcha Enferm. 2021; 42(esp): e20200281doi: https://doi. org/10.1590/1983-1447.2021.20200281.

16. Backes MTS, Carvalho KM, Santos EKA, Backes DS. New coronavirus: what does nursing have to learn and teach in times of a pandemic?. Rev Bras Enferm. 2020;73(Suppl 2):e20200259. doi: http://dx.doi.org/10.1590/0034-7167-2020-0259. 